\title{
The Various Misconceptions of Bipolar Disorder in Barbados
}

\author{
Article by Marlo A.R Browne \\ Master of Public Health, Texila American University, Barbados \\ E-mail: ricobro@hotmail.com
}

\begin{abstract}
Bipolar disorder, also known as manic depressive illness, is a complex, chronic and severe mental health disorder that is characterized by episodes of mania, depression or a combination of both. The study seeks to examine the plethora of current and prevalent misconceptions perceived by members of the general population within the Caribbean country of Barbados. Different research methodologies were employed for the purpose of the study, including the conduction of the survey, which illustrates the general misconceptions, held by the Barbadian public, as well as interviews with local mental health professionals to discredit these misapprehensions. Four hundred people completed the survey. Results indicate that there is a percentage of the population which understood the disease, but which believes that effective knowledge dissemination was the key to the increase of awareness and the reduction of the misconceptions, as well as the fact that females were susceptible to the disorder, especially during the ages of 25-29.
\end{abstract}

Keywords: Bipolar Disorder, Misconceptions, Barbados

\section{Introduction}

Bipolar disorder, also known as manic depressive illness, is a complex, chronic and severe mental health disorder that is characterized by episodes of mania, depression or a combination of both. Craddock and Jones (1999) describe it as a complex genetic disorder where the primary feature is an incorrigible disturbance in mood that fluctuates between periods of euphoria or jubilation, which is termed as mania, to periods of severe depression which usually affects thinking and behavior patterns and may include some psychotic symptoms like delusions and hallucinations. They elaborate further by defining bipolar disorder as an episodic illness, suggesting that full recovery can occur within episodes. Sajatovic(2005) agrees with this view, by defining bipolar disorder as a chronic psychiatric illness, that has distinguishable and recurrent features of mania, hypomania and depression and contributes to the global economic burden of disease, due to the fact that the actual treatment cost of the disease may exceed a total of $\$ 7$ billion. She stresses the importance of bipolar disorder as a global public health concern by providing statistics which indicate its prevalence could lie within the range of 1 and $2 \%$ and that approximately $73 \%$ of patients experience a relapse within the duration of 5 years. Therefore, she cites it as the sixth highest cause of medical disability among the US population, between the ages of 15 and 44. She also notes that it caused a staggering unemployment rate of $60 \%$ among the patients that were examined. Although the American Health Association sub-classifies bipolar disorder into two recognizable types, which have been conveniently named Bipolar I and Bipolar II disorder respectively, in their book The Diagnostic and Statistical Manual of Mental Disorders (1952), there are in actuality several types, including cyclothymic disorder. Craddock and Jones (1999) define bipolar I disorder, as classified by the DSMIV, as a form of the illness in which the patient experiences extreme manic or mixed episodes. The manic episodes may last for duration of seven days. Sajatovic(2005) implies that persons with this form of the disease are about 3 times as susceptible and 7 more times susceptible to alcohol and drug abuse and dependence respectively, comparable to the general population. She extends her point by expressing the view that patients with bipolar disorder are 26 more times susceptible to panic disorders, as well as 8 more times likely to experience obsessive compulsive disorder. In 
contrast, bipolar II disorder is defined as the form of the disease where the patient undergoes more depressive episodes and only milder forms of mania, which are often referred to as hypomania. Gitlin(2006), in his article, states that the depressive episodes in bipolar II patients, greatly outweighed the hypomanic episodes, boasting a ratio of 39:1. In Barbados, research proposes that the average amount of healthy years lost per 100,000, due to bipolar disorder has increased from 1990 by a rate of $2.9 \%$. This research also suggests that the peak age range of those affected by the disorder, falls within the productive years of 25-29 and that women were more susceptible to it, with the amount of healthy years lost estimated to be about 310 per 100,000 .

\section{Purpose of study}

The purpose of this research is to effectively evaluate the plethora of current and prevalent misconceptions of bipolar disorder that are perceived by members of the general population within the Caribbean country of Barbados. Through this article, I hope to educate them with the knowledge so that the social stigma that is heavily placed on people who suffer from mental illnesses, including bipolar disorder, is reduced. In Barbados, there has been a certain proclivity to perpetuate the belief that all moody behaviors are associated with bipolar disorder. This is in fact, a blatant myth and can be attributed to the lack of knowledge about the chronic medical condition. This is also due to the person's inherent laziness to access the available information so that they can be able to educate themselves thoroughly about the disease. Ghaemi and Dally (2015) agree with this, by stating that there are people who believe that since mood liability can be an important criterion in the definition of borderline personality, then it can precipitate to bipolar and other personality disorders. They elaborate, through the usage of previous research, conducted by Kraepelin, in the 1900s, to compare mood liability to a fever. They convey this comparison by stating that since a fever is a symptom of pneumonia, but is non-specific, then it is unable to be used in the nosology of pneumonia. Through this approach, the view is imparted that mood liability is like the fever of psychiatry, showing that although it may be prevalent with several conditions, it does not illustrate the true representation of the diseases that may be present. Another common myth that needs to be rectified is that bipolar disorder is an endogenous process (Hlastala et al, 2000). This can also be attributed to the lack of thorough education and research.

\section{Theoretical framework}

\section{Bipolar disorder and age detect ability}

There is a recurrent fallacy among the general population that mental health practitioners are unable to detect and diagnose bipolar disorder until the patient reaches the age of 18 . Sajatovic(2005) disputes this by saying that there was no specific correlation between age and the mental health disorder, but it was noted that the apogee for its onset existed within the range between 15 to 19 years of age. She provides further evidence to debunk this myth through the information obtained from two different sources. The first piece of evidence from a survey conducted by the National Depressive and Manic-Depressive Association (NDMDA) elucidated that approximately 59\% of the present patients with bipolar disorder were said to experience their first symptoms, either through childhood or at the adolescent stage. The second piece of evidence came through another study which suggested that the onset of disease could occur in patients, aged 60 years and older. Some researchers imply that the illness should be treated seriously and should not be mistaken for children going through their normal trials and tribulations. Instead, they believe that children with bipolar disorder are often unable to sleep, as well as performing to their full potential in school. Almeida et al (2002) compares the two sets of patients, and they are of the view that patients who experience the disease during adulthood are strongly associated with organic brain disease, while those who have the early onset are associated with a hereditary origin. Post et al (2010) support this view by stating that onset of the disease was extremely common in children and this eventually led to these patients experiencing a significant increase in the severity and the 
duration with which they encountered manic and depressive episodes in comparison to those patients who developed the disorder during adulthood. They attributed this to the fact that the patients whose onset emerged during childhood, received a lag in their treatment.

\section{Bipolar disorder and quality of life}

There is a popular vantage point that people with bipolar disorder are able to lead normal lives and be contributing members to the society, as long as they receive the proper treatment. However, research has shown that bipolar disorder often contributes to the concept of functional impairment. Bowden (2005) provides evidence of this through the utilization of information from various studies. In one study, conducted by the NDMDA, it was emphasized that the amount of people who had occupational difficulties was an embarrassing 88\%. Another study conducted in the United States, highlighted the social stigma that was associated with bipolar disorder. Through the perception of the danger associated with mental disorders, there was a drastic decline in the amount of paid employment by as much as $40 \%$. A third study involved a follow up period of six months, where it showed that although $80 \%$ of the patients were symptom free or mildly symptomatic, only $43 \%$ of them were fully employed. Of that percentage, all of them were hospitalized for a manic episode. Sajatovic(2005), supports this by saying that in another study, it was discovered that approximately $64 \%$ of the patients observed failed to commit to long-term relationships, while $65 \%$ of the respondents had problems with their children. However, she attributes this to either of two facts, a) That most of the patients go untreated and/or b) There is an occurrence of comorbidity. She contends the view that bipolar patients are unable to lead functional lives by implying that the authors of the study had limited knowledge and that as time passed and more information became available, then it became possible for the impairment of these individuals to decrease. Through that view, it can be proposed that this observation is not entirely untrue.

\section{Violent behaviors associated with bipolar disorder}

Another popular misconception is the perception that everyone who has bipolar disorder is extremely violent. This is in fact, not true. Researchers concur that while people with mental disorders may tend to exhibit bursts of violent behavior, it can be attributed to other factors and not the disorder itself. Vann (2010) agrees with this and cites factors such as substance abuse and emotional stress as contributors to violence. She insinuates that substance abuse is more prevalent in people with mental illnesses and this increases the incidence of episodes, which predisposes to the violent act. She also states that dealing with the loss of a loved one can increase emotional stress, acting as the trigger to a violent outburst in bipolar patients. Sajatovic (2005) also implies that bipolar patients are more susceptible to substance abuse by stating that as much as 41 and $46 \%$ had drug and alcohol abuse and dependence respectively. It can also be implied that the more addicted or dependent an individual is to a particular substance, the more violent he or she may become, if he or she is unable to attain that substance. This also detracts the image that mental illnesses, including bipolar disorder, are associated with violent or dangerous behavior.

\section{Suicide and bipolar disorder}

In contrast to the previous view, people with bipolar disorder are more of a danger to themselves. Vann (2010) supports this claim by saying that although bystanders may fear for their lives when interacting with people who have this condition, the real damage is dealt to the person living with the disease. She elaborates this by suggesting that people with bipolar disorder are 9 times more likely to take their own lives than those of their peers. Sajatovic (2005) provides further evidence of this when she implies that according to one study, the suicide rate associated with bipolar disorder cases was significantly higher than any other axis I psychiatric disorders, boasting an odds ratio of 6.2. Using another study, she proposed that suicidal attempts in bipolar patients were as much as $50 \%$, with $35 \%$ of these attempts 
Texila International Journal of Public Health

Volume 4, Issue 4, Dec 2016

resulting in hospitalization. She concluded her point by showing that the total rate of completed suicide was $20 \%$.

\section{Methodology}

\section{Survey of views that the barbadian public has about bipolar disorder}

A survey design was employed in the study. Employees from four different businesses, as well as pedestrians, were asked to speak about their beliefs, regarding disease etiology, functional impairment, behavioral patterns, as well as any treatment that was associated with the disorder. They were also asked if they believed that people with bipolar disorder were indeed crazy. The employees from businesses included managers, administration, janitors and every possible employee that was present, including those that were physically disabled. All views were documented for the purpose of this article, which amassed to a total of 400 persons.

\section{Interviews with nurses and psychiatrists from the psychiatric hospital}

Several interviews were conducted to debunk the myths that were associated with the mental disorder. The questions that were asked included ones on the prevalence of the disease in the country, age of onset of the disease, violent behaviors associated with the disease, gender most affected by the disease, comparison of it with other Caribbean countries as well as the overall care of all mental health patients that lived within the Psychiatric Hospital. All results were documented, either written or graphically for the purpose of this article.

\section{Presentation of results}

\section{Survey results}

Results from the survey served as an indication that the Barbadian public had contrasting views when it came to the issue of bipolar disorder. Approximately $31 \%$ of the respondents were uncertain about the disease, asking if it exists. Of that percentage, one was bold enough to state that he did not have the time to think about that. Another $15 \%$ of the respondents believe that there is a lack of empirical and statistical evidence to authenticate the validity of the prevalence of the disorder. One respondent even suggested that people with this disease are possessed, citing a verse from the Bible as his reference. The verse, Ephesians 6, verse 12, depicts the view that human beings are in a battle that is not of flesh, but they are fighting against cosmic forces who reign over darkness. Therefore, the respondent is implying that these forces are responsible for the changes in the patients' moods and behavioral patterns. Another respondent was very articulate in his response. He said that he believed that it was a period of emotional change and propounded that there was a comprehensive list of people who held esteemed positions that had the disorder. He continued by saying that there was a switch in moods, going from a period of extreme happiness to a state of depression. Approximately 54\% of the respondents had an indication of the existence of the disease. Half of that percentage cited emotional stress as one of the etiological factors, utilizing the stories of family members who had the disease to speak about functional impairment and behavioral patterns. One of these respondents painted a vivid picture where she described her niece as holding a steady job at the bank, where she interacted vivaciously with her coworkers, but when she left the establishment, she was a different person. She stated that her niece believed that people, with the exception of her workmates were enemies. Another one of these respondents said that one of her family members experienced a bad ending to a relationship, which served as the emotional trigger for the onset of the disorder. She proposed that the major misconception that Barbadians possess was the perception that the afflicted individuals are crazy. However, in actuality, once they receive the appropriate treatment, they can lead fully functional lives. Another female respondent suggested that the awareness of the disease needs to be increased in Barbados because the general population views it as a joke and they fail to understand the severity of the disorder. She emphasizes that a social stigma is still 
associated with the disorder and that the people with the illness needs specialized treatment so that they will be able to live normal lives. Another female respondent corroborated this view and felt empathy for the bipolar patients. She said that it takes a lot of emotional strength to endure that condition and she always admires their determination.

\section{Results from the interviews with the mental health professionals}

All of the mental health professionals indicated that bipolar disorder was a mental disorder in which there are manic and depressive episodes that are coordinated, but occur at sporadic rates. They were very tight-lipped on the conditions with which the patients are treated in the Psychiatric Hospital, saying that they received optimal care. The answers to the other questions, such as the prevalence of the disease, age of onset and comparison with other countries are illustrated below.

Table 1: Bipolar disorder relative to other mental and substance use disorders (Barbados)

\begin{tabular}{|l|l|l|}
\hline Cause of Harm & Daily Rate per 100 000 & Change (1990-2013) \\
\hline Depressive Disorders & 1031 & $5 \%$ \\
\hline Anxiety Disorders & 581.5 & $0 \%$ \\
\hline Schizophrenia & 227.7 & $17 \%$ \\
\hline Bipolar Disorder & 173 & $3 \%$ \\
\hline Drug Use Disorders & 158.8 & $12 \%$ \\
\hline $\begin{array}{l}\text { Other Mental and Substance } \\
\text { Use Disorders }\end{array}$ & 143.4 & $12 \%$ \\
\hline
\end{tabular}

Through this table, it is shown that the three most enervating mental health disorders in Barbados during 2013 were depressive disorders, anxiety disorders and schizophrenia respectively. The major change, related to increased prevalence of the disease was schizophrenia, which boasted an increase of $17 \%$ over the last decade. The number of reported cases of bipolar disorder only increased by 3\% during this period and the mental health professionals theorized that due to this miniature increase of cases, an inverse proportion of awareness of the disease was generated during that period. As a result, Barbadians came up with many misconceptions through ignorance. They also proposed that although various demographic groups are affected differently, the statistics might also vary in relation to age.

Table 2: Bipolar Disorder Impact Relative to Other Locations in the Caribbean

\begin{tabular}{|l|l|l|}
\hline Location & Daily Rate per 100,000 & Change (1990- 2013) \\
\hline Cuba & 176.8 & $5 \%$ \\
\hline Trinidad and Tobago & 173.7 & $17 \%$ \\
\hline Barbados & 173 & $3 \%$ \\
\hline The Bahamas & 171.5 & $13 \%$ \\
\hline Antigua and Barbuda & 164.1 & $3 \%$ \\
\hline Saint Lucia & 164 & $20 \%$ \\
\hline $\begin{array}{l}\text { Saint Vincent and the } \\
\text { Grenadines }\end{array}$ & 162.2 & $19 \%$ \\
\hline
\end{tabular}

From this table, it can be seen that Barbados is the country with the third highest number of cases in the Caribbean, although the overall change in cases over the last decade is minimal. This provides further evidence to support the view that bipolar disorder is a serious mental health condition in Barbados, which is contrary to the general consensus of the average Barbadian.

The medical health professionals also stated that the health burden of the disease was at its pinnacle when both sexes reached the ages of 25-29, but argued that women were more susceptible. They said that the average amount of healthy years lost for women during that age range was about 310 per 100,000, while it was about 258.9 per 100,000 in men. They also 
said that the disease was least harmful during the ages of 10-14 in both sexes, with the amount of healthy years lost being 30.2 per 100,000 and 24.4 per 100,000 in females and males respectively. In comparison to the global burden of disease, the average amount of healthy years lost in Barbados were 173 per 100,000, while in the world, it was about 130 per 100,000 .

\section{Discussion}

The statistics provided by the mental health professionals presents verification of the disease, debunking the myth that there is a lack of statistical data. However, I believe that this information should be made more accessible to the general public so that they would not be basking in their ignorance and formulating these bizarre misconceptions. This is supported through the response given by one individual to the survey, where he suggested that the afflicted individuals are possessed. With increased availability and accessibility to this information, the average Barbadian could become more cognizant, which would lead to a drastic decline to the social stigma that prevails in the small Caribbean country. People with mental illnesses are shunned and treated as if they are deranged. Subsequently, they tend to be discriminated against or exploited, either through diminished job opportunities or the failure to maintain effective relationships with significant others. One respondent was quoted as saying that he could not deal with those bipolar individuals, because one minute they are good, then in the next minute, they "freak out".

Shamsaei et al (2013) zone in on the severity of the disorder by proposing that even the individuals that receive the optimal mental health care are still susceptible to relapses and therefore have trouble keeping jobs and establishing effective social relationships with others. They estimate that the overall lifetime prevalence of the disorder, falls within the range of 1 and $2 \%$. They also support the view that Sajatovic held, by implying that bipolar disorder was one of the world's most debilitating conditions and that it can affect anyone, irrespective of nationality, race or socio-economic status. They also suggest that family members of the afflicted individuals, as well as the primary caregivers can experience a similar level of social stigma as the bipolar individual, through association. Their research shows that family members experienced feelings of isolation and shame and that they were often criticized by other family members as well as by the mental health practitioners. This situation is also prevalent in Barbados. I have seen several instances where family members are blatantly humiliated by other people due to the fact that they are related to an individual with a mental disorder. Shamsaei et al (2013) are also of the view that bipolar patients are deprived of the ability to acquire satisfactory housing conditions. Evidence of this is also seen in Barbados. I am aware one incident where one particular person was denied the opportunity to rent an apartment because she had a mental history. The landlord told the prospective tenant that he was unable to rent the apartment to her, because she was crazy and that he does not want any 'loony goon' around his premises.

I could also see the hurt of some of the family members of the patients of bipolar disorder who had participated in the survey. The respondent whose niece worked at the bank talked to me in detail after the survey was completed. She expressed her concern that a good proportion of the Barbadian population are ignorant to mental disorders and she wanted to avoid her niece having any unnecessary confrontation with anyone in the workplace. She informed me that to interact with a bipolar individual was difficult, because you had to carefully monitor the mercurial changes in their behavior, and not just classify them as "mood swings." She also said that she was tired of the perception that Barbadians hold that every individual that experience a sudden change in mood was bipolar. She concurred with the view that I had, in which I believe that only through proper research and understanding of the disorder, can the misconceptions of the disease be obliterated. Ghaemi and Dally (2015), believe that the problem stems from the lack of precision in the definition of bipolar spectrum conceptions. They use previous research to attribute this to the fact that prior definitions involve vague and 
over-inclusive language. They stress that emphasis should be placed on mood temperaments, not mood liability, since this is an important part of the bipolar spectrum.

Another female Barbadian respondent also implied that there was a small percentage of the Barbadian population who realized that mental disorders, including bipolar disorder, can be inherited. She concurred with me that a lot was not known about its etiology. Ghaemi and Dally (2015) describe this as the biopsychosocial errors that are associated with borderline personality, and indirectly bipolar disorder. They propose that the etiology of this disorder is almost completely genetic, with an approximation of about $80 \%$ of the heritability based on previous twin studies and that psychosocial factors rarely contribute to disease etiology, since only about $50 \%$ of all personality traits are genetic in bipolar disorder.

The medical professionals also quashed the delusion that bipolar disorder was only detectable at age 18. From the results, it can be seen that the disorder was detected from as early as 10 years old in both sexes, but it was severely heightened at ages 25-29, with the average amount of healthy years lost being 284.4 per 100,000 in 2013. It can also be suggested that the reason why Barbadians do not consider bipolar disorder to be a significant problem was the fact that there are not a lot of reported cases of it in Barbados, so the information would not be as widespread as depressive disorders. If you refer to table 1 , you would notice that the daily rate of depressive disorders in Barbados was 1031 per 100,000 in 2013, in contrast to bipolar disorder, which was a meager 173, indicating that the ratio of daily cases of depressive disorders to bipolar disorders was almost 6:1. Therefore, there was a lot more information about depression than bipolar disorder, so Barbadians do not consider it to be a serious problem, although, as shown in table 2, Barbados has the third highest prevalence rate of the disease in the Caribbean, as measured in 2013.

\section{Conclusion}

Bipolar disorder is simply not an appellation that was created by scientists. It is a chronic, recurrent and remitting mental illness, in which the manifestational criteria for classifying the disease include separate periods of mania and depression. Mania includes excessive behavior and inflated self-esteem as well as the perception held by the individual that they do not require sleep, whereas depressive episodes include changes in sleep patterns, appetite, low self-worth and suicidal thoughts and plans. Without efficient knowledge dissemination, a variety of misconceptions developed within the country of Barbados. This research article serves as the platform, where several of those fallacies were discredited and the factual information came to light. Again I reiterate that the information needs to be properly distributed, so that the levels of ignorance and social stigmas that accompany mental illnesses can be reduced. For a country that boasts of its population's literacy, the number of misconceptions that occurred were appalling. Through the use of descriptive statistics, it was shown that although it is not as serious as other depressive disorders in Barbados, bipolar disorder is still pretty significant. It is a condition that needs to be treated seriously, so that the afflicted persons can receive the appropriate and timely treatment, in order that they can lead successful lives. From the survey, it is shown that they can live productive lives, but certain aspects of their lives, such as social interaction, still need to be addressed.

\section{Acknowledgement}

I would like to thank Ms. Faye Boyce for her help in compiling the data for this article. I would also like to thank the respondents of the survey, as well as the mental health professionals that participated in the interviews. Without them, this article would not be possible. 
Texila International Journal of Public Health

Volume 4, Issue 4, Dec 2016

\section{References}

[1] Almeida, Oswaldo P and Fenner, Stephen. Bipolar Disorder: Similarities and Differences Between Patients With Illness Onset Before and After 65 Years of Age in International Psychogeriatrics, Volume 14, Issue 3, pp. 311-322 (2002).

[2] American Psychiatry Association. Diagnostic and Statistical Manual of Mental Disorders (1952)

[3] Bowden, Charles L. Bipolar Disorder and Work Loss (2005)

[4] Craddock, Nick and Jones, Ian. Genetics of Bipolar Disorder in J Med Genet, Issue 36, pp. 585-594 (1999)

[5] Ghaemi, S Nassir and Dally, Shannon. The Bipolar Spectrum: Conceptions and Misconceptions (2015)

[6] Gitlin, M. Treatment- resistant Bipolar Disorder in Molecular Psychiatry, Issue 11, pp. 227-240 (2006)

[7] Hlastala, Stephanie A, Frank, Ellen, Kowalski, Jeanne, Sherrill, Joel T, Tu, Xin M, Anderson, Barbara and Kupfer, David J. Stressful Life Events, Bipolar Disorder and the "Kindling Model" in Journal of Abnormal Psychology, Volume 109, Issue 4, pp. 777-786 (2000)

[8] Post, Robert M, Leverich, Gabriele S, Kupka, Ralph W, Keck Jr, Paul E, McElroy, Susan L, Altshuler, Lori L, Frye, Mark A, Luckenbaugh, David A, Rowe, Michael, Grunze, Heinz, Suppes, Trisha and Nolen, William A. Early Onset Bipolar Disorder and Treatment Delay are Risk Factors for Poor Outcome in Adulthood in The Journal of Clinical Psychiatry, Volume 71, Issue 7, pp. 864-872 (2010)

[9] Sajatovic, Martha. Bipolar Disorder: Disease Burden in Am J Manag Care, Volume 11, pp. S80S84 (2005)

[10] Shamsaei, Farshid, Kermanshahi, Sima Mohammed Khan, Vanaki, Zohreh and Holtforth, Martin Grosse. Family Care-giving in Bipolar Disorder: Experiences of Stigma in Iran J Psychiatry, Volume 8, Issue 4, pp.188-194 (2013)

[11] Vann, Madeline. Are People with Bipolar Disorder Dangerous? (2010) 